\title{
Variability of olfactory threshold and its role in assessment of aging
}

\author{
JOSEPH C. STEVENS and AASHISH D. DADARWALA \\ John B. Pierce Laboratory, New Haven, Connecticut \\ and Yale University, New Haven, Connecticut
}

\begin{abstract}
Olfactory thresholds of elderly persons (over 65 years) average one to two orders of magnitude higher than those of young adults (under 30 years). Past studies reveal enormous spreads (typi. cally about three orders of magnitude) of individual thresholds within each age group and extensive overlap between the two groups-enough to question how typically decline in sensitivity characterizes the individual aged person. The present study shows that much of the observed overlap is misleading, because the brief threshold tests usually administered tend to exaggerate individual differences. A more representative assessment of an individual's threshold (for 1-butanol) was achieved by averaging the thresholds from two to eight separate short tests, spread over 4 days. The spread of each group's thresholds (12 young and 12 elderly subjects) narrowed strikingly as the number of tests averaged increased from one to four; further tests accomplished no additional narrowing of spread. Based on a single test, thresholds of young and elderly overlapped in the usual way; but based on four or more tests, thresholds of young and elderly overlapped little or not at all. The outcome (1) argues that decline in smell sensitivity seems to be, after all, a common feature of aging, and (2) sheds light on the sources of variability of sensory thresholds.
\end{abstract}

A weakened sense of smell is a feature of getting older. Three psychophysical indications of this are: (1) increase in average absolute threshold for a variety of compounds (for reviews see Murphy, 1986; Schiffman, 1979; Van Toller, Dodd, \& Billing, 1985), (2) decrease in strength of suprathreshold odors (Stevens, Bartoshuk, \& Cain, 1984; Stevens \& Cain, 1985, 1986; Stevens, Plantinga, \& Cain, 1982), and (3) decline in ability to name odors (Doty et al., 1984; Schemper, Voss, \& Cain, 1981; Stevens \& Cain, 1987). The present study addresses the following question: Does loss of absolute sensitivity mark the individual aged person?

When all three of the above measures were combined to rank overall individual olfactory performance, it appeared to Stevens and Cain (1987) that almost all persons over 70 years of age showed impairment compared with young adults. Threshold data of Venstrom and Amoore (1968), Van Toller et al. (1985) and, recently, of Cain et al. (1993), show that weakening appears in the fourth decade and progresses thereafter. These and other facts led Cain and Stevens (1989) to conclude that olfactory weakèning is a normal, gradual, and pervasive feature of aging. Ship and Weiffenbach (1993) recently came to the same conclusion. On the other hand, Almkvist, Berglund, and Nordin (1992) uncovered no difference in the thresh-

This research was supported by Grant AG-04287 from the National Institute on Aging. The authors thank Matthew Q. Patterson, Julianne Hoffman, Enrique Cometto-Muñiz, William S. Cain, and Lawrence E. Marks for technical and editorial assistance. Address correspondence and reprint requests to J. C. Stevens, John B. Pierce Laboratory, 290 Congress Avenue, New Haven, CT 06519. olds (to pyridine) of young and elderly persons denoted as "successfully aged," which they defined as elderly persons spared of cognitive and medical deficiencies.

Numerous studies have reported that the average elderly person has a significantly higher threshold than the average young person. But why, if olfactory loss is a feature of normal aging, do the thresholds of young and elderly subjects overlap so much? As an example, Figure 1 (from Stevens, Cain, Schiet, \& Oatley, 1989) displays thresholds to pyridine for 63 young and 77 elderly subjects. The average threshold for the elderly subjects was about 10 times higher than that for the young. There is, however, enough overlap of the thresholds to question whether threshold losses typify individual aging; that is, whether threshold is a reliable index of aging among individuals.

Figure 1 seems to indicate that many old persons perform as well as or even better than the average young person! To evaluate what this means, it is helpful to consider test-retest reliability and to distinguish the terms "sensitivity" and "threshold," remembering that they are often used in different ways. Thresholds may be thought of as more or less transient operations by which to gauge underlying sensitivity. Sensitivity is a more enduring property of a sense modality. According to this view, threshold might be operationally defined in a variety of ways; for example, as that level of a stimulus detected a certain proportion of the time. This level itself may, however, fluctuate over a sufficient time frame. A "threshold" based on a relatively short sample of responses may, therefore, differ in size from a "threshold" based on a longer sample or on several short samples over time. According to this conception, "sensitivity" may be approximated but 


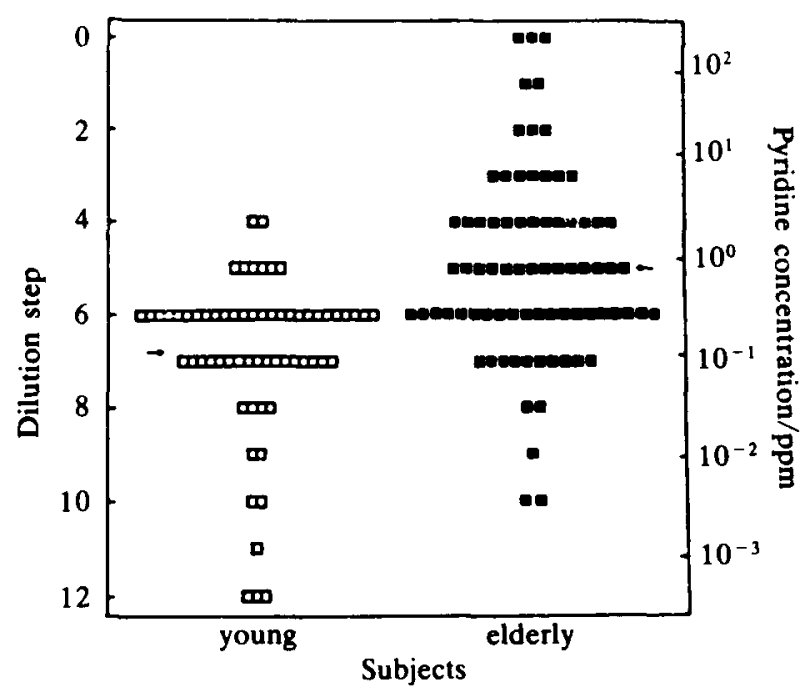

Figure 1. Absolute thresholds to pyridine for 63 young (16-35 years) and 77 elderly (68-89 years) subjects. The arrows show the mean thresholds for the two groups (from Stevens et al., 1989).

never fully defined by any given measurement of threshold, much as a baseball player's batting average over any particular number of innings will only approximate his hitting ability. This, too, oversimplifies reality because, over time, sensitivity itself, like batting ability, can change, as when a person grows older, or tired, or when the sense organ is overloaded by adaptation. In aging and other "clinical" contexts, threshold has, for practical reasons, usually been assessed in short tests. These are imperfect representations of sensitivity.

In the present study, we argue that, when appropriately measured, threshold can furnish a more reliable indicator of individual aging than has hitherto appeared. A key factor is the nature of variability of thresholds, not only among but also within subjects, from one testing situation to another. The olfactory scientist knows only too well that threshold varies tremendously from one "normal" person to another. As Amoore (1980) summarized, "about $96 \%$ [i.e., plus and minus two standard deviations] of the population have sensitivities between 16 and $1 / 16$ of the normal [i.e., 256 to 1]' (p. 33). This figure may be conservative. Variability radically depends on the particular compound, for reasons that are not fully understood. For example, Punter (1983), tested a large group of young students on 58 different compounds, and found that the standard deviation of the individual thresholds (expressed in $\log _{2} \mathrm{ppb}$ ) varied from 0.78 for toluene (lowest) to 5.31 for tetrachloromethane (highest), with a median of 2.165. To appreciate what these numbers mean and to compare them to Amoore's summary figure of $256 / 1$, they translate to $10 / 1,2,500,000 / 1$, and $400 / 1$ for the low, high, and median standard deviations. For Punter's three different assessments of butanol (the odorant used in the present study), they translate to $50 / 1,750 / 1$, and $10,000 / 1$. Numbers on this order turn up routinely (see, e.g., Brown,
Maclean, \& Robinette, 1968; Jones, 1957; Rabin \& Cain, 1986; Stevens \& Cain, 1987; Yoshida, 1984). To explain (1) why intersubject variability itself varies so much across compounds, and (2) why it is so high compared with other sense modalities constitutes two major challenges to the student of olfaction.

Another challenging dimension is the discovery that, for any single compound, the variability of an individual subject tested from one time to another is only scarcely smaller than the intersubject variability of a group of cohorts. A person who appears to be one of the most sensitive subjects on one day may appear to be one of the least sensitive on the next. This is reflected in Punter's (1983) low test-retest correlations from young subjects (18-24 years) for a subset of 11 of his 58 compounds-a median $r$ of .40 , thus accounting for only $16 \%$ of the variance. The correlations comparing subjects across compounds was also very low-median .28 of 1,081 coefficients (pairwise comparisons of 47 compounds), accounting for only $8 \%$ of the variance (i.e., a subject's relative threshold appears to be minimally consistent across compounds). Correlation coefficients of similar small size characterize all the studies on threshold variability cited above. Small coefficients characterize our findings here, too (see below).

Variability of the individual's threshold highlighted Stevens, Cain, and Burke's (1988) measurement of 20 thresholds in each of 3 subjects and each of three compounds. Of each set of 20,2 were measured on each of 10 different days. Variability on the same day was the same as that from day to day (important to the present study) and was in both cases very high-from 6 to 11 ternary dilution steps across compounds and subjects. Butanol threshold varied by an average of 6.3 steps across subjects $(1,000 / 1$ range), and the other two compounds showed similar results. In contrast, for each compound, mean threshold was practically the same for all 3 subjects. Thus, the average of several short tests may yield a more stable impression of individual sensitivity and furnish a reliable index of individual olfactory aging.

Rabin and Cain (1986) and Cain and Gent (1991) advocated frequent testing over time, arguing that the usual brief tests overestimate individual differences. Rabin and Cain's conclusion that "high person-to-person variability in olfactory sensitivity may be largely an illusion" (p. 284) foreshadows the present study. Cain and Gent also recognized that intrasubject variability may approach intersubject variability. The "interval of uncertainty"that is, the concentration span over which performance ranges from just above chance $(50 \%$ correct on twoalternative forced choice) to $100 \%$ correct-was, in their study, 1,000/1 for both individuals and the group.

\section{METHOD}

\section{Subjects}

Each of 12 young ( 7 female, 5 male, 19-26 years, $M=22.4$ ) and 12 elderly persons ( 7 female, 5 male, 69-91 years, $M=76.5$ ) took part in four 1-h (or shorter) test sessions on 4 different days, 
over 1-2 weeks (except 1 subject took 4 weeks). (Because of gross and persistent inconsistency across and within sessions, 1 elderly subject had to be replaced.) The subjects earned $\$ 6$ per session and a $\$ 6$ bonus after the last session ( $\$ 30$ total). The young subjects were recruited, on a first come first served basis, through advertisements on bulletin boards at Yale University, and were tested in the laboratory; the elderly subjects were recnuited in the same way, but were tested at two senior citizens' centers. All the subjects lived independently at home. All of the 12 young subjects rated their health as good; 10 elderly subjects also rated their health as good; 2 as average.

\section{Stimulus Materials}

The task was detection of 1-butanol vapor by a double-blind, twoalternative, forced-choice procedure. On each trial, the subject had to decide which of a pair of polypropylene "shampoo" bottles $(250 \mathrm{ml})$, one containing butanol dissolved in deionized water (DHOH) and the other containing DHOH only (the blank), emitted the stronger smell when the vapor headspace was ejected (by squeezing the bottle) through the spout, just below the nostrils. A procedure of this kind is often used in clinics and laboratories (e.g., Amoore, 1980; Cain, Reid, \& Stevens, 1990; Cain et al., 1993; Rabin \& Cain, 1986; Stevens \& Cain, 1987; Stevens et al., 1989; Stevens, Cain, \& Weinstein, 1987). A test set consisted of 13 concentrations separated by threefold dilution steps from 0 (stock solution) to 12 (weakest solution) and a blank; there were two such sets, alternated on successive trials to allow the headspace to equilibrate after ejection. The stock solution (zero step) was $4 \%$ $(v / v)$ butanol in $\mathrm{DHOH}$, which corresponds to concentration in air (headspace) of 3,100 ppm as determined by gas chromatography. In the present study, stimulus levels are usually cited as a step level from 0 to 12; corresponding parts per million are proportional to liquid concentration.

\section{Psychophysical Method}

For a threshold, a subject made 30 successive forced-choice trials by the "step method," an adaptive method devised by Simpson (1989) to give a relatively efficient and unbiased estimate of threshold in under 40 trials. Unlike most adaptive procedures (Wetherill \& Levitt, 1965), which vary by zero or one level from one trial to the next, the step method allows for multistep changes. These occur frequently near the start of a track but give way to single and zero step changes as the track stabilizes. To avoid confusion, one should remember that "concentration step" and "dilution step" (conventional units of olfactometric strength) are negatively related; thus, an increase of one concentration step (threefold increase of parts per million) corresponds to a decrease of one dilution step. Simpson's "step method" refers to concentration or stimulus-level steps, not to dilution steps.

The $n$th concentration step in the track was chosen by a protocol programed in BASIC in an NEC laptop or Macintosh computer. Essentially, after each trial, it computes an estimate of the threshold concentration step, which in turn serves as the level for the next trial. It does this by selecting the concentration step with the lowest cumulated error term (sum of squared error, or SSE). Whenever the subject chooses correctly, the SSE for that concentration step and for all higher concentration steps is incremented by $1^{2}$ (which equals one); when the choice is correct, the SSE for that step and for all lower steps is incremented by $1^{2}$ times a weighting factor of four. (The size of the weighting factor determines the criterion level for correct forced-choice responding. In the present application, a weighting factor of four yielded approximately $80 \%$ correct responding.) By this rule, several steps frequently tie for SSE; ties are resolved by taking a weighted average of the tied steps and rounding it to the nearest whole step.
Several alternative procedures present themselves for deciding on the threshold from a track. One can, for example, average some portion of the track-say, the last 5, 10, or 20 trials. Or, one can select that level associated with the lowest SSE for the $n$th +1 trial ( $n=30$ in the present experiment). These all gave remarkably comparable outcomes. The measure adopted, minimal SSE, was used also by Simpson (1989).

\section{Test Design}

Each subject served in four test sessions (about 45 min each) on separate but not necessarily consecutive days. Each session consisted of instructions, a threshold run of 30 trials, an 8-10 min rest, and another threshold run of 30 trials. Thus, each subject produced eight thresholds altogether. Trials occurred every $30 \mathrm{sec}$, so each threshold took $15 \mathrm{~min}$.

\section{RESULTS}

Figure 2 plots all 192 thresholds separated by age group. Three features emerge (all seen in earlier studies). (1) Threshold varied greatly across subjects, spanning all 13 stimulus levels (a concentration ratio of nearly $1,600,000 / 1)$. The elderly subjects proved more variable than the young, perhaps, as Stevens and Cain (1987) speculated, because they age physiologically at different rates. The elderly subjects spanned 10 steps $(60,000 / 1$ in concentration); the young spanned seven steps $(2,200 / 1)$. (2) The average young subject was clearly more sensitive than the average elderly subject-by 3.56 steps (50/1). (3) Thresholds for the young and elderly subjects overlapped by five steps (240-fold). The degree of overlap as well as the mean difference will doubtless vary with the exact ages of the subjects, psychophysical method, and sampling error (see Discussion). But the main point is clear: Single-test estimates of threshold do not reliably pinpoint a subject's age group.

A quite different picture unfolded, however, when each subject's eight thresholds across sessions were averaged and then replotted in Figure 2. Three main features emerged here. (1) The variability of these averages or "composite" thresholds was much smaller than the individual ones-a span for the young subjects of 3.5 steps (reduced from 7) and for the elderly subjects of 4.5 steps (reduced from 10). (2) The mean difference between young and elderly composites, the same as for the individual thresholds, was 3.5 steps $\left[t(22)=6.6, p<10^{-6}\right]$. (3) Unlike the individual thresholds, the composites separate out well by age.

Separate analyses of each of the eight tests revealed that the mean difference between the young and old subjects and the exact degree of overlap did, of course, vary from test to test, thereby demonstrating that any single comparison of brief tests should be taken with a grain of salt. Each test did yield a statistically reliable mean difference $(p<.001)$, but the difference obtained varied from a low of 2.61 dilution steps (18-fold gas concentration range) for Test 7 to a high of 3.72 steps (60-fold range) for Test 6 . Such fluctuation, even in the very same subjects 


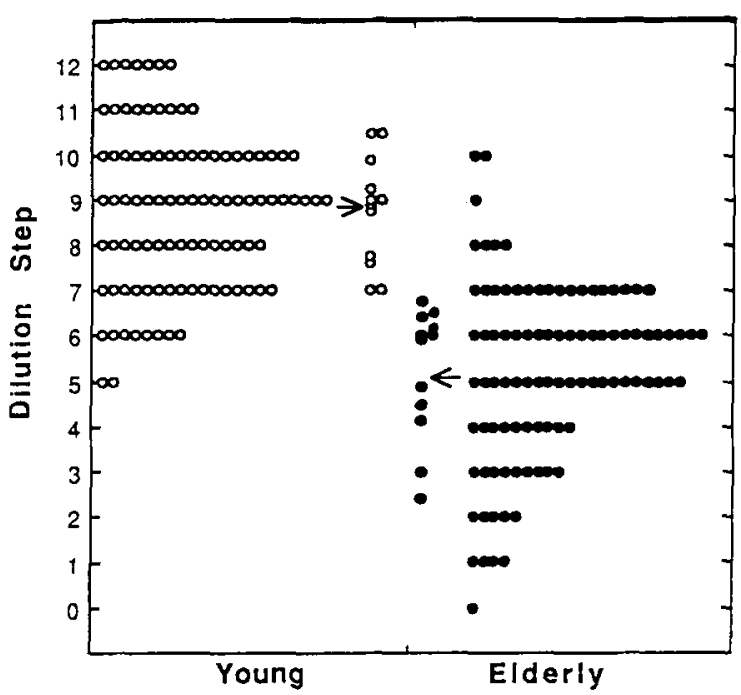

Figure 2. The open circles at the extreme left show all 96 individual thresholds for the 12 young subjects; the filled circles at the extreme right show all 96 individual thresholds for the 12 elderly subjects. The circles in the middle show the average of the 8 thresholds of each subject. Arrows mark the means for the young and the elderly subjects. tested by the same psychophysical method, warns against taking too seriously the exact size of an age-related threshold difference obtained in a single sampling of brief tests.

Figure 3 shows, for the old and young subjects: (1) the mean thresholds and standard deviations for Tests 1-8 taken separately (panels A and C), and (2) mean thresholds and standard deviations of thresholds constituting individual cumulative (composite) averages for Tests 1,2 , 3 , and so on, up to and including all eight tests (panels $B$ and $D$ ). The figure reveals three points. (1) Mean thresholds for individual sessions (panel A) and cumulatively averaged sessions (panel B) tended to decline (mean dilution step rises, by about $2 / 3$ ternary step, or $2 / 1$ in parts per million) with more testing, thereby confirming Cain and Gent (1991). (2) Variability (standard deviation) fluctuated a bit from test to test (panel C), but did so unsystematically with more testing. The standard deviations of the cumulatively averaged thresholds, however, fell strikingly from Tests 1-4 (panel D). Neither the means nor the standard deviations of these cumulative thresholds changed after Test 4 (second day). These observations indicate that (3) nothing was gained in reliability by testing more than four times.

(A)

(B)
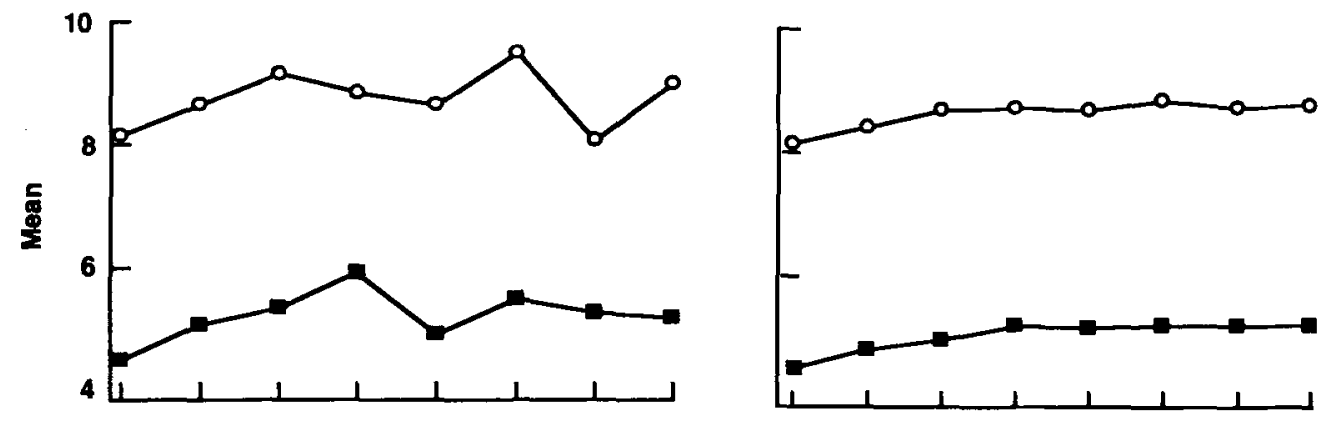

(C)

(D)
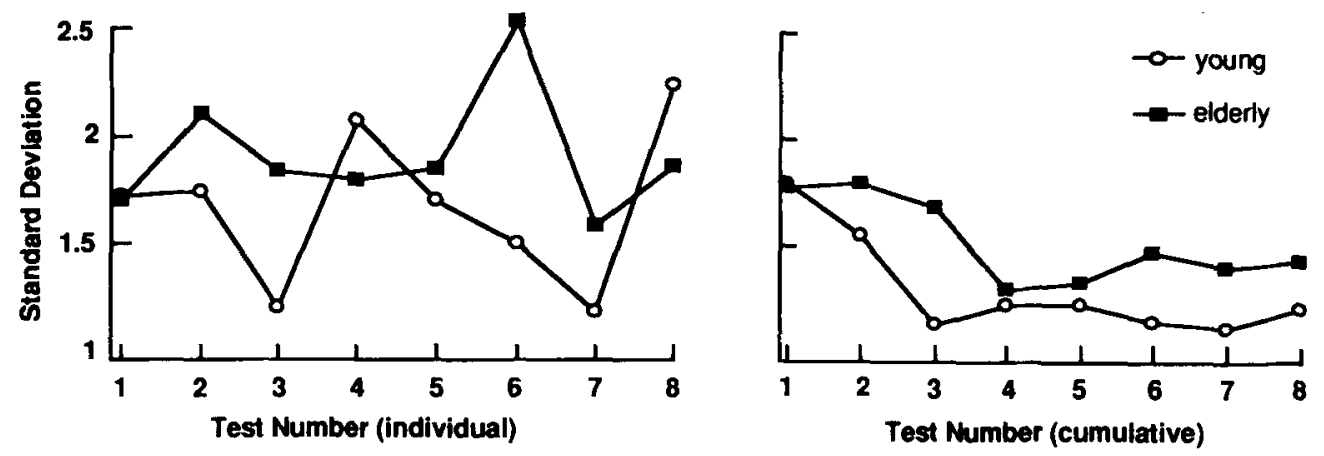

Figure 3. Means (panels $A$ and B) and standard deviations (panels $C$ and D) for individual threshold tests separately (panels $A$ and $C$ ) and for cumulative averages of tests, from one to eight inclusive (panels $B$ and D), as designated in the text. Circles $=12$ young subjects; squares $=12$ elderly subjects. 


\section{Correlations Among Tests}

The reduction in variability that was effected by averaging the tests and the consequent disentanglement of the data from the young and elderly subjects could occur only because the test-retest correlation between any two tests is low, as noted above. This is true not only for the present data, but also for those of other researchers cited heremost notably Punter's (1983), because of its broad scope and forced-choice methodology. To the extent that testretest correlation approaches 1.0 , nothing is to be gained by repeated testing. To the extent that it approaches zero, each test of an individual is like testing different individuals.

Interpair correlations came to about $r=.4$, interestingly enough whether for test pairs obtained in the same day's session or for pairs relating tests on different days, and, whether for young or for elderly subjects. Of the $56 \mathrm{rs}$ relating all possible pairs for the young and elderly subjects separately, 52 turned out to be positive in sign; of these, 19 were significant at $p<.05$. A weak, positive correlation thus prevailed for both the young and elderly subjects. The mean $r$ correlation for same-day and different-day comparisons both came to .41 .

When evaluating interpair correlations (whether intraor interodorant), one must take into account the makeup (homogeneity) of the subjects' ages. When all 24 thresholds of the young and elderly subjects for each test were combined, they yielded a median $r$ of .723 for the 28 possible pairings of the eight tests. This compares closely with the interodorant correlations reported by Cain and Gent (1991) for persons of heterogeneous ages between 22 and 59 years, and with interodorant correlations (median of $12 r \mathrm{~s}=.60$ ) reported by Patterson and Stevens (1993) in a study of odor mixtures (see below).

\section{Analysis of Variance}

All 192 individual thresholds were subjected to analysis of variance (ANOVA) comparing the variables of age, different days, different tests within the same day, and their various interactions. Of these, only age proved significant $\left(p<10^{-10}\right)$. Of prime interest here was the finding that the variance associated with different days was on the same order as the variance associated with different tests on the same day, confirming Stevens et al.'s (1988) study of pairs of thresholds obtained on 10 different days. The present study indicates that this may be true even when a pair is separated by only 8-10 min.

The change from one threshold to the next in the same test session was as likely to be positive as negative; this proves that we deal here with no simple sensory adaptation. It is presently speculative whether to view the changes as spontaneous discontinuities in real sensitivity or as a kind of sampling error in a purely random stochastic process. The possible role of rest periods is discussed below in the light of another study from our laboratory (Patterson, 1992).

\section{DISCUSSION}

Variability across subjects and within the same subject from test to test seems at first glance to be a formidable obstacle against characterizing individual olfactory sensitivity; customary brief threshold scores prove poor indicators of an individual's age. On the other hand, much can be gained by averaging a few such tests. In the present study, averaging allowed a virtually complete separation of all the 12 young subjects from the 12 elderly subjects; unaveraged, the thresholds showed considerable overlap.

The present results also confirm and extend those of Stevens et al. (1988) concerning the sources and nature of variability. That study made clear that an individual's variability over time is almost as great as the variability among individuals. This finding, together with those cited above, can be summarized in six sources of variability.

1. Chemical compound. The intersubject variability is higher for some odorants than for others. In Punter's (1983) survey of 58 compounds, the standard deviation ranged over five to six orders of magnitude.

2. Among individuals. This depends radically on the compound, but for the median compound in Punter's survey of young subjects it was about $400 / 1(=1 S D)$. However, a large portion of this variability may be attributable to within-individual variation.

3. Within individuals. Based on measurements by Stevens et al. (1988) and test-retest coefficients from other experiments (typical $r$ s about .4), variability within subjects is only slightly smaller than among individuals tested in brief tests. That the test-retest correlation is significantly larger than zero argues, however, for a sensitivity factor characterizing the individual. This is discussed below.

4. Age. Various studies place this figure between one and two orders of magnitude between young adulthood and old age. For butanol, the figures range from about 30- to 100-fold (Cain \& Gent, 1991, 100-fold; Cain et al., 1990, 50-fold; Cain et al., 1993, 35-fold; Patterson, 1992, 30 -fold; present study, 50 -fold). The exact difference will depend on sampling error (as illustrated by the variation stated above, among the eight tests of the present study), the particular compound (Stevens \& Cain, 1987; Van Toller et al., 1985; Wysocki \& Gilbert, 1989), the age makeup of the samples (rarely fully matched), and the psychophysical method.

5. Practice. Practice over 3 to 4 days was explored by Rabin and Cain (1986) and Cain and Gent (1991). The latter showed an average decline of $25 \%$ in threshold over four test sessions on different days, in reasonable agreement with the present study, which yielded an average decline to about one half over the first four tests. Cain and Gent also noted that a subject tends to become more consistent, as evidenced by increases in their interodorant correlations. This was also evident in Patterson's (1992) interodorant correlations, which were higher for the second day's test (median $r=.71$ ) than for the first (median $r=.52$ ).

6. Miscellaneous factors. As discussed by Stevens et al. (1988), these include temperature and humidity (Grundvig, Dustman, \& Beck, 1967), stimulus delivery system (Punter, 1983), style of sniffing (Laing, 1983), and psychophysical method. It is tempting but seemingly futile 
to invoke these sources to explain the first three sources listed here, because although each may contribute, their total impact is diminutive by comparison. We simply do not understand why threshold varies so much among compounds, people, and the same person over time.

\section{Stable Individual Differences}

The above facts about sources of variability leave room for real, long-term sensitivity differences among individuals, even after the effects of age have been partialled out. The persistent test-retest correlation near $r=.4$ indicates a sensitivity factor, as does the spread of the young and elderly subjects' average thresholds (composites), as shown in Figure 2. Some idea of the degree of the persistent sensitivity is furnished by comparing two average thresholds for each subject, one of which is the average of four of the tests, the other the average of the other four tests. The median $r$ for all possible pairings of such quads of tests (35 $r$ s per age group) was .775 (.73 for the young subjects and .79 for the elderly). In other words, thresholds based on four tracks are respectably reliable, at least compared with thresholds based on a single track (for which $r$ was about .4).

Because in the present study same-day and different-day variances were approximately equal (as well as same-day and different-day correlations), it would appear that a brief rest and a whole day or more of rest may have equivalent effects on absolute threshold. In other words, we might obtain a more reliable picture of a subject's sensitivity from several short samples of trials separated by brief intervals of rest than from a like number of trials unbroken by rests; future research will explore this possibility.

\section{Number of Trials and Rest Breaks}

There is some reason to suppose that the total number of stimulus trials (though doubtless very important) may not be the only thing at stake in accounting for the benefit of repeated testing. That rest periods may play a special role was suggested by data from this laboratory by Patterson (1992) on odor mixtures (see also Patterson \& Stevens, 1993). Subjects gave thresholds, one after the other without significant breaks between them, for three different compounds and their mixture; on a subsequent day, they were retested on the same stimuli. The mixture had a lower threshold than did its components (i.e., there was summation). However, interest centers here on the result that the variance of thresholds obtained back-to-back was about one half that for thresholds obtained on separate days; this is signified by an average $r^{2}$ that was about twice as large for back-to-back thresholds as for separateday thresholds. Same-session (back-to-back) correlations relating the various pairs of chemical stimuli averaged .50 (after partialling out the correlation between threshold and age); different-session partial correlations averaged .34close, incidentally, to Punter's (1983) correlation for young subjects (median $r=.28$ ) relating the 1,081 pairs of 47 compounds. (As noted above, because thresholds depend on age, the interodorant and intraodorant correlations are larger if age is not partialled out from a sample of heterogeneous ages. In Patterson's study, data from young and elderly subjects combined yielded median $r s$ of .60 [same-session, back-to-back correlations] and .49 [different-day correlations], which roughly approximate the values reported in both the present study and Cain \& Gent, 1991.)

That correlations obtained among tests in proximity (no break) were substantially higher than those obtained on different days suggests that two brief tests separated by a rest may represent a subject's sensitivity better than one test of twice the number of trials unbroken by rest. The extent to which this is true and, more broadly, the extent to which the right scheduling of trials and rests can minimize total testing time for a reliable individual threshold, remains to be worked out. The present study suggests that four short tests separated by rests of about $8 \mathrm{~min}$ (or a little over $1 \mathrm{~h}$ of testing time) might suffice. The search for an effective, practical testing schedule is a future goal, and the present study offers leads on how to achieve this.

The present results warn us, also, against staking too much on a single, brief test of threshold. Repeated measures have taught us that the difference between young and elderly people is far more clear-cut than has hitherto been apparent, and they bolster the view that weakened sensitivity is a "normal" feature of aging. The present study also serves to highlight some of the components of variability and to furnish a rough characterization of their relative sizes. Eventually, a more exact mathematical model of these components may be feasible, which could apply to sensory thresholds in general. Because it is so variable, at least on the surface of things, the olfactory threshold may be a good point of departure.

\section{REFERENCES}

Almkvist, O., Berglund, B., Nordin, S. (1992). Odor detectability in successfully aged elderly and young adults (Tech. Rep. No. 74, pp. 1-12). Stockholm: Stockholm University, Department of Psychology.

AMOORE, J. E. (1980). Properties of olfactory system. In F. H. Suchomel \& J. W. Weatherly (Eds.), Institute of gas technology symposium on odorization (pp. 31-35). Chicago: Institute of Gas Technology.

Brown, K. S., Maclean, C. M., \& Robinette, R. R. (1968). The distribution of the sensitivity to chemical odors in man. Human Biology, 40, 456-472.

CAIN, W. S., \& GENT, J. F. (1991). Olfactory sensitivity: Reliability, generality, and association with aging. Jourmal of Experimental Psychology: Human Perception \& Performance, 17, 382-391.

Cain, W. S., Reid, F., \& STEvens, J. C. (1990). Missing ingredients: Aging and the discrimination of flavor. Journal of Nutrition for the Elderly, 9, 3-15.

CaIN, W. S., \& Stevens, J. C. (1989). Uniformity of olfactory loss in aging. Annals of the New York Academy of Sciences, 561, 29-38.

Cain, W. S., Stevens, J. C., Nickou, C. M., Giles, A., Johnston, I., \& GarCia-Medina, M. R. (1993). Life-span development of odor identification, leaming, and olfactory sensitivity. Manuscript submitted for publication.

Doty, R. L., Shaman, P., Applebaum, S. L., Giberson, R., SikSORSKI, L., \& ROSENBERG, L. (1984). Smell identification ability: Changes with age. Science, 226, 1441-1443. 
Grundvig, J. L., Dustman, R. E., \& Beck, E. C. (1967). The relationship of olfactory receptor stimulation to stimulus-environmental temperature. Experimental Neurology, 18, 416-428.

JONES, F. N. (1957). An analysis of individual differences in olfactory thresholds. American Journal of Psychology, 70, 227-232.

LAING, D. G. (1983). Natural sniffing gives optimum odour perception for humans. Perception, 12, 99-117.

MurPhy, C. (1986). Taste and smell in the elderly. In H. L. Meiselman \& R. S. Rivlin (Eds.), Clinical measurement of taste and smell (pp. 343-371). New York: Macmillan.

Patterson, M. Q. (1992). Olfactory thresholds: Detection of a mixture and its components. Unpublished senior thesis, Yale University, New Haven, CT.

Patterson, M. Q., \& Stevens, J. C. (1993). Detection of olfactory mixtures and their components. Manuscript submitted for publication.

PunTER, P. H. (1983). Measurement of human olfactory thresholds for several groups of structurally related compounds. Chemical Senses, 7, 215-235.

Rabin, M. D., \& CaIN, W. S. (1986). Determinants of measured olfactory sensitivity. Perception \& Psychophysics, 39, 281-286.

SCHEMPER, T., Voss, S., \& CAIN, W. S. (1981). Odor identification in young and elderly persons: Sensory and cognitive limitations. Journal of Gerontology, 36, 446-452.

Schiffman, S. (1979). Changes in taste and smell with age: Psychophysical aspects. In J. M. Ordy \& K. Brizzee (Eds.), Sensory systems and communication in the elderly (Vol. 10, pp. 227-246). New York: Raven.

Ship, J. A., \& Weiffenbach, J. M. (1993). Age, gender, medical treatment, and medication effects on smell identification. Joumal of Gerontology: Medical Sciences, 48, M26-M32.

Simpson, W. A. (1989). The step method: A new adaptive psychophysical procedure. Perception \& Psychophysics, 45, 572-576.

Stevens, J. C., Bartoshuk, L. M., \& Cain, W. S. (1984). Chemical senses and aging: Taste versus smell. Chemical Senses, 9, 167-179.
Stevens, J. C., \& Cain, W. S. (1985). Age-related deficiency in the perceived strength of six odorants. Chemical Senses, 10, 517-529.

Stevens, J. C., \& Cain, W. S. (1986). Smelling via the mouth: Effect of aging. Perception \& Psychophysics, 40, 142-146.

Stevens, J. C., \& Cain, W. S. (1987). Old-age deficits in the sense of smell as gauged by thresholds, magnitude matching, and odor identification. Psychology \& Aging, 2, 36-42.

Stevens, J. C., Cain, W. S., \& Burke, R. J. (1988). Variability of olfactory thresholds. Chemical Senses, 13, 643-653.

Stevens, J. C., Cain, W. S., Schiet, F. T., \& Oatley, M. W. (1989). Olfactory adaptation in old age. Perception, 18, 265-275.

Stevens, J. C., Cain, W. S., \& Weinstein, D. E. (1987). Aging impairs the ability to detect gas odor. Fire Technology, 23, 198-204

Stevens, J. C., Plantinga, A., \& Cain, W. S. (1982). Reduction of odor and nasal pungency associated with aging. Neurobiology of $\mathrm{Ag}$ ing, 3, 125-132.

Van Toller, C., Dodd, G. H., \& Billing, A. (1985). Ageing and the sense of smell. Springfield, IL: Thomas.

VENSTROM, D., \& AMOORE, J. E. (1968). Olfactory threshold in relation to age, sex, or smoking. Journal of Food Science, 33, 264-265.

WeTheriLl, G. B., \& LeviTt, H. (1965). Sequential estimation of points on a psychometric function. British Journal of Mathematical \& Statistical Psychology, 18, 1-10.

WysockI, C. J., \& GILbERT, A. N. (1989). National Geographic smell survey. In C. Murphy, W. S. Cain, \& D. M. Hegsted (Eds.), Nutrition and the chemical senses in aging. Annals of the New York Academy of Sciences, 561, 12-28.

YoshiDA, M. (1984). Correlational analysis of detection threshold data for "standard test" odors. Bulletin of the Faculty of Science \& Engineering of Chuo University, 27, 343-353.

(Manuscript received October 20, 1992; revision accepted for publication February 22, 1993.) 